\title{
LESÃO TRANSITÓRIA NO ESPLÊNIO DO CORPO CALOSO EM CRIANÇA EPILÉPTICA COM GLIOMA CEREBRAL DE BAIXO GRAU
}

\author{
Vicente José Assencio-Ferreira', Marcelo Lucci Mussi², \\ Vinicius Monteiro de Paula Guirado 3 , José Carlos Esteves Veiga ${ }^{4}$
}

\begin{abstract}
RESUMO - Trata-se do relato de caso de menino de sete anos com crises epilépticas parciais complexas secundárias à presença de glioma de baixo grau em região fronto-temporal esquerda, cuja ressonância magnética evidenciou, lesão transitória focal do esplênio do corpo caloso. A revisão bibliográfica através de pesquisa no database MedLine resultou no encontro de descrição de lesão transitória do esplênio do corpo caloso foi relatada em três estudos anteriores, em pacientes portadores de epilepsia. Portanto, a lesão transitória observada no corpo caloso desta criança, provavelmente, tem correlação com as crises epilépticas do lobo temporal e não à presença do glioma de baixo grau cerebral.
\end{abstract}

PALAVRAS-CHAVE: corpo caloso, glioma de baixo grau, epilepsia, ressonância magnética, drogas antiepilépticas.

\begin{abstract}
Transient lesion in the splenium of the corpus callosum in epileptic child with cerebral low grade glioma
ABSTRACT - We report on a seven years-old boy with complex partial seizures and the presence of low grade glioma in left fronto-parietal region. The magnetic resonance imaging showed focal non-hemorrhagic lesion in the splenium of the corpus callosum. The description of the transient lesion in the splenium of the corpus callosum was related in three previous studies, in patients with epilepsy. Thus, the observed transient focal lesion in the splenium of the corpus callosum of this child, probably, has correlation with to prolonged focal partial seizures and not to the presence of glioma low grade.
\end{abstract}

KEY WORDS: corpus calossum, low-grade glioma, epilepsy, magnetic resonance imaging, antiepileptic drugs.

As crises epilépticas do lobo temporal são definidas por serem parciais complexas e eletrencefalograma (EEG) com atividade irritativa na região temporal. A etiologia, freqüentemente, pode ser determinada através de estudos por neuroimagem e o substrato mais encontrado é a perda neuronal e gliose da região hipocampal, classicamente denominada de esclerose mesial temporal. O corpo caloso é constituído por cerca de 300-800 milhões de fibras inter-hemisféricas provenientes fundamentalmente do córtex cerebral. As conexões, na sua maioria, ocorrem entre áreas homólogas dos córtices associativos sensoriais, pré-motores e áreas motoras suplementares, obedecendo a topografia específica. Na região do esplênio do corpo caloso, as fibras inter-hemisféricas são provenientes do córtex temporal superior, temporal inferior e córtex justa-estriado. A revisão da literatura sobre lesões do corpo caloso levou ao encontro de três relatos com descrição de altera- ções idênticas às observadas no presente caso, em 16 pacientes com epilepsia do lobo temporal ${ }^{1-4}$. Os mecanismos fisiopatológicos ainda não estão adequadamente identificados, tendo sido levantada a hipótese de toxicidade a vigabatrina/fenitoína ${ }^{2}$ e de tratar-se de disfunção axonal transitória dependente das crises epilépticas ${ }^{3}$.

O objetivo deste relato é chamar a atenção par a que o encontro para alteração do esplênio do corpo caloso no estudo de neuroimagem por ressonância magnética (RM) em crianças com crises epilépticas parciais pode ser uma manifestação transitória.

\section{CASO}

Menino, branco, com sete anos e sete meses, primeiro filho de pais não consangüíneos, nascido de parto cesárea sem intercorrências e com desenvolvimento neuropsicomotor normal. Iniciou com crises epilépticas há um ano e dois meses, do tipo parciais complexas, com posterior ge-

Disciplina de Neurologia e Neurocirurgia do Hospital Universitário Faculdade de Medicina da Universidade de Taubaté, Taubaté SP, Brasil (UNITAU): 'Doutor em Medicina (Neurologia) pela Universidade de São Paulo (USP), Colaborador Assistente Doutor da UNITAU; ${ }^{2}$ Residente do $2^{\circ}$ ano de Neurologia da UNITAU; ${ }^{3}$ Residente do $4^{\circ}$ ano de Neurocirurgia do Hospital Santa Isabel de Clínicas (HOSIC) Taubaté, SP, Brasil; 'Professor Doutor e Chefe da Disciplina de Neurocirurgia da UNITAU. 


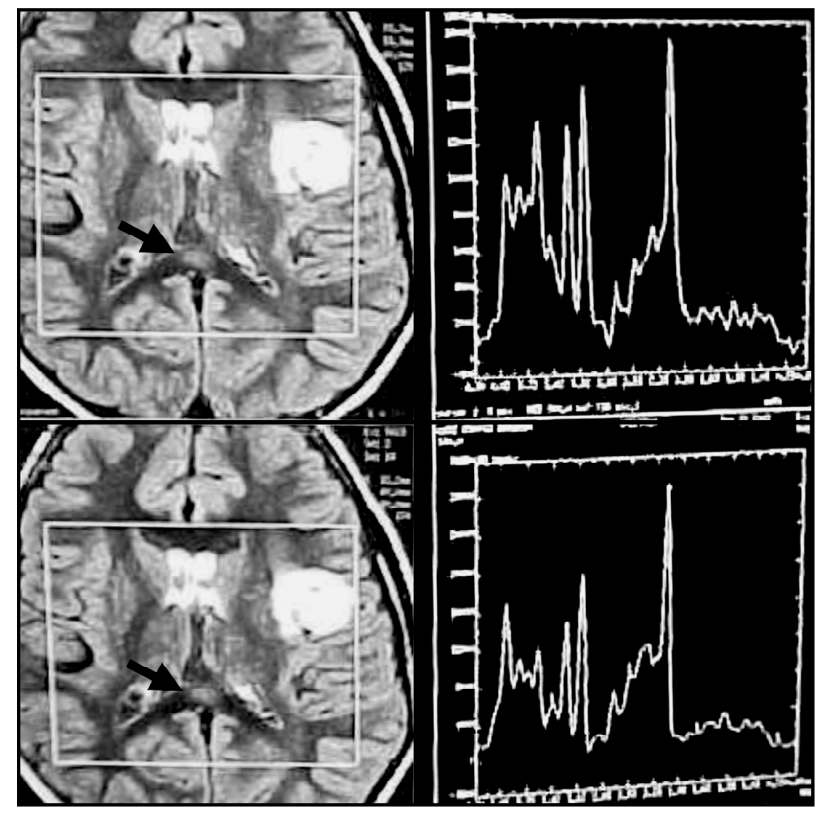

Fig 1. À esquerda: Ressonância magnética em apresentação $T 2$ com duas lesões distintas: lesão tumoral parietal esquerda e, na região do esplênio do corpo caloso, imagem arredondada de hipersinal (seta). À direita - Espectroscopia de prótons da lesão tumoral com discreta elevação do pico de mioinositol, discreta re dução do pico de $\mathrm{N}$-acetil aspartato e ausência de elevação significativa do pico de colina em relação ao de creatina, que caracterizam tumor de baixo grau.

neralização, que ocorriam preferencialmente durante o sono. Foi obtido o controle das crises após nove meses, quando se adequou a dose de carbamazepina. Tomografia computadorizada (TC), evidenciou área hipodensa parieto-temporal esquerda RM, mostrou presença de lesão tumoral em região inferior e lateral do giro précentral esquerdo e porção ântero-superior da ínsula ipsilateral. A espectroscopia de prótons desta lesão foi compatível com a hipótese de glioma de baixo grau. Outro achado do exame foi uma imagem arredondada de hiperintensidade de sinal em T2 e na seqüência flair, comprometendo a região central do esplênio do corpo caloso (Fig 1).

Optou-se pela conduta expectante e controle do desenvolvimento tumoral. Após seis meses foi realizada nova RM e não mais se observou hipersinal do esplênio do corpo caloso, enquanto que a lesão tumoral permaneceu inalterada.

O presente relato de caso foi avaliado e aprovado pelo Comitê de Ética em Pesquisa da Universidade de Taubaté.

\section{DISCUSSÃO}

A lesão transitória do corpo caloso observada em pacientes com epilepsia é raramente relatada na literatura e tem sua fisiopatologia ainda não identificada. No primeiro relato, em $1996^{1}$, foram descritos sete pacientes com epilepsia do lobo tem- poral e a lesão foi interpretada como edema focal transitório dependente da passagem de atividade elétrica anormal através do corpo caloso, quando da generalização secundária da crise epiléptica. Após três anos, outros seis pacientes tinham sido relatados, todos com epilepsia parcial complexa do lobo temporal; em apenas um caso observou-se generalização secundária. Foi levantada a hipótese de tratar-se de toxicidade das drogas antiepilépticas (vigabatrina e fenitoína), pois as lesões desapareceram após a retirada destes medicamentos ${ }^{2}$.

Em 2001, três novos casos foram relatados, sendo que em dois a alteração do EEG estava localizada no lobo temporal; no último a crise epiléptica foi descrita como não classificada, pois apresentava crises parciais e generalizadas e o EEG não demonstrou alterações. A hipótese fisiopatológica aventada para a lesão transitória do esplênio do corpo caloso foi da multifatorialidade ${ }^{3}$.

O presente caso foi classificado como epilepsia parcial complexa, tanto pela apresentação clínica como pelo EEG com atividade irritativa temporal esquerda. Assim, de 17 pacientes relatados (incluindo o presente relato), 16 tiveram a epilepsia classificada como de lobo temporal; no único caso em que não foi possível a classificação, o paciente apresentava crises parciais seguidas ou não de generalização secundária.

No caso aqui relatado, foi observada a presença de edema transitório na região correspondente a somatotopia das fibras inter-hemisféricas de conexão dos córtices temporais (esplênio do corpo caloso). Assim, é possível supor que esta lesão dependa das descargas eletroquímicas exageradas que ocorreram quando da crise epiléptica temporal prolongada que a criança apresentou.

A presença de lesão transitória de hipersinal em T2 e flair no esplênio do corpo caloso, observada na RM do presente caso, muito provavelmente, está associada às crises epilépticas temporais prolongadas e não à presença do glioma de baixo grau.

\section{REFERÊNCIAS}

1. Chason DP, Fleckenstein JL, Ginsburg ML, Mendelsohn DB, Mathews D. Transient splenial edema in epilepsy: MR imaging evaluation. Presented at the annual meeting of the American Society of Neuroradiology; Seattle, WA: June 21-27,1996.

2. Kim SS, Chang K-H, Kim ST, et al. Focal lesion in the splenium of the corpus callosum in epileptic patients: antiepileptic drug toxicity? AJNR 1999;20:125-129.

3. Polster T, Hoppe M, Ebner A. Transient lesion in the splenium of the corpus callosum: three further cases in epileptic patients and a pathophysiological hypothesis. J Neurol Neurosurg Psychiatry 2001;70:459-463.

4. Verstichel P, Degos JD. Syndromes de déconnexion interhémisphérique. Encycl Med Chir Paris: Elsevier: Neurologie 17036-C-10, 2000. 